\title{
Suppression of host adaptive immune responses by Neisseria gonorrhoeae: role of interleukin 10 and type 1 regulatory $T$ cells
}

\author{
Y Liu ${ }^{1}$, W Liu ${ }^{2}$ and MW Russell ${ }^{1}$
}

Infection with Neisseria gonorrhoeae triggers an intense inflammatory response characterized by an influx of neutrophils in the genital tract, yet natural gonococcal infection does not induce a state of protective immunity. Our previous studies in a mouse model of $\boldsymbol{N}$. gonorrhoeae infection demonstrated that transforming growth factor- $\beta$ (TGF- $\beta$ ) is involved in the suppression of adaptive immunity by this organism, but complete inhibition of TGF- $\beta$ activity only partially reverses N. gonorrhoeae-mediated suppression of T helper type 1 (Th1) and Th2 responses. In this study, we show that $N$. gonorrhoeae strongly induced the production of interleukin (IL)-10 and type 1 regulatory T (Tr1) cells. Blockade of IL-10 and Tr1 cell activity enhanced both Th1/Th2-dependent adaptive immune responses and Th17-governed innate responses to $N$. gonorrhoeae. Treatment of mice with anti-IL-10 antibody during gonococcal challenge led to faster clearance of infection and induced protection against secondary infection, with the generation of circulating and vaginal anti-gonococcal antibodies. Our results suggest that inhibition of IL-10 and Tr1 cells affords a new approach to the treatment of gonorrhea and facilitates the development of specific protective immunity.

\section{INTRODUCTION}

Gonorrhea is the second most common sexually transmitted disease. The latest World Health Organization report estimates that $>100$ million new gonococcal infections occur worldwide every year, ${ }^{1}$ and the increasing resistance of Neisseria gonorrhoeae to even the latest generations of antibiotics is becoming problematic for effective treatment strategies. ${ }^{2}$ Ascending gonococcal infections occur frequently, particularly in women, leading to fallopian tube scarring, pelvic inflammatory disease, infertility, and risk of ectopic pregnancy, which can be lifethreatening. ${ }^{3}$ Epidemiological and clinical studies provide strong evidence that gonorrhea significantly increases the risk of acquisition and transmission of HIV.,

$N$. gonorrhoeae typically triggers an intense inflammatory response characterized by an influx of neutrophils into the genital tract, yet natural gonococcal infection does not induce a state of specific protective immunity. ${ }^{5,6}$ Individuals with gonorrhea are usually not protected from reinfection, although one study reported partial protection against the same serovar of N. gonorrhoeae. ${ }^{7}$ This apparent lack of an adaptive immune response to $N$. gonorrhoeae probably contributes to the continuing prevalence of this sexually transmitted infection and challenges the development of a vaccine against it. The conventional working hypothesis holds that $N$. gonorrhoeae can evade host immune defenses by multifactorial strategies, including continuous changes in its surface antigenic structure, resistance to complement-mediated bacteriolysis, and possibly the production of immunoglobulin A1 (IgA1) protease., ${ }^{5-10}$ However, increasing evidence indicates that $N$. gonorrhoeae as a highly adapted pathogen has evolved specialized mechanisms to proactively suppress specific immune responses and promote growth and persistence in the host. For example, it has been demonstrated that N. gonorrhoeae opacity (Opa) proteins are able to bind carcinoembryonic antigen-related cellular adhesion molecule (CEACAM)-1 on activated human $\mathrm{CD} 4 \mathrm{~T}$ cells and downregulate their activation and proliferation. ${ }^{11}$ Recently, Zhu et al. ${ }^{12}$ reported that $N$. gonorrhoeae could inhibit both human and mouse antigen-dependent CD4 T-cell

\footnotetext{
${ }^{1}$ Department of Microbiology and Immunology and Witebsky Center for Microbial Pathogenesis and Immunology, University at Buffalo, Buffalo, New York, USA and ${ }^{2}$ Department of Pediatrics, Digestive Diseases and Nutrition Center, University at Buffalo, Buffalo, New York, USA. Correspondence: Y Liu (yingruli@buffalo.edu or yingru_ubc@yahoo.com) 
proliferation through interactions with host antigen-presenting dendritic cells.

Although it has been recognized that $N$. gonorrhoeae possesses the capacity to modulate host immune responses, the underlying mechanisms remain to be elucidated. Furthermore, comprehension of how this can be manipulated to generate protective adaptive immunity against the organism is limited. Our previous studies in a mouse model of gonococcal infection have demonstrated that $N$. gonorrhoeae elicits $\mathrm{T}$ helper type 17 (Th17) responses, which are involved in the influx of neutrophils to the genital tract as well as the recruitment of other innate defense mechanisms. ${ }^{13}$ By contrast, N. gonorrhoeae can selectively suppress Th1 and Th2 activity of mouse CD4 T cells, and induction of transforming growth factor- $\beta$ (TGF- $\beta$ ) has a critical role in these differential effects. ${ }^{14,15}$ Blockade of TGF- $\beta$ diverts the pattern of host immune responses to $N$. gonorrhoeae and enhances specific protective immunity against the pathogen. However, we found that complete inhibition of TGF- $\beta$ activity only partially reverses $N$. gonorrhoeae-mediated suppression of Th1 and Th2 cell proliferation and typical cytokine production. ${ }^{15}$ These results suggest that other unknown mechanisms also have an important role in the inhibitory effect of $N$. gonorrhoeae on Th1/Th2-mediated adaptive immune responses.

Interleukin (IL)-10 is a regulatory cytokine produced by a variety of immune cells, including activated $T$ cells, monocytes/ macrophages, B cells, dendritic cells, and mast cells, ${ }^{16}$ and it has a major role in suppressing immune and inflammatory responses and maintaining specific $\mathrm{T}$-cell tolerance in both humans and mice. ${ }^{17}$ Type 1 regulatory $\mathrm{T}(\mathrm{Tr} 1)$ cells are one type of induced regulatory $\mathrm{T}$ (Treg) cells, which inhibits Th1, Th2, and Th17 immunity through the production of immunosuppressive cytokines, mainly IL- $10{ }^{18} \operatorname{Tr} 1$ cells arise in the periphery when naive $\mathrm{CD} 4{ }^{+} \mathrm{T}$ cells are activated by tolerogenic antigen-presenting cells (APC) in the presence of IL-10. ${ }^{19}$ Therefore, the biological functions of IL-10 and Tr1 cells are closely related to each other. IL-10 is not only responsible for the regulatory effect of $\operatorname{Tr} 1$ cells but is also fundamental for their generation. Accumulating evidence indicates that IL-10 and Tr1 cells have a key role in regulating mucosal immune activation, for example, in the maintenance of gut immune homeostasis and tolerance to food antigens and enteric microbiota. ${ }^{20,21}$ In addition, IL-10 and Tr1 cells are exploited by many pathogens at mucosal sites to evade protective immunity, including Bordetella pertussis and Porphyromonas gingivalis. ${ }^{22,23}$ However, the potential role of IL-10 and Tr1 cells in genital tract infections has not been explored.

In this study, we show that, both in vitro and in vivo, $N$. gonorrhoeae strongly induced the production of IL-10 and Tr1 cells, which are critically involved in the suppression of adaptive immunity by the organism. Blockade of IL-10 and Tr1 cell activity significantly increased Th1, Th2, and Th17 responses to $N$. gonorrhoeae and resulted in the generation of immune memory in a mouse model of vaginal gonococcal infection. Furthermore, our results revealed that inhibition of IL-10 in combination with inhibition of TGF- $\beta$ had an additive effect in promoting protective immunity against N. gonorrhoeae.

\section{RESULTS \\ N. gonorrhoeae elicits abundant production of IL-10 and Tr1 cells in vitro}

To determine whether N. gonorrhoeae is capable of inducing IL-10 and Tr1 cells, we incubated mouse iliac lymph node (ILN) cells with N. gonorrhoeae (FA1090) in serum-free medium for various time periods. After 4 days, mouse lymphocytes stimulated with $N$. gonorrhoeae produced extremely high levels of IL-10, but not of Th1- or Th2-type cytokines, such as IL-12p70 or IL-4 (Figure 1a). Flow cytometric analysis showed that IL-10 was produced in multiple immune cell types, including $\mathrm{CD}_{4}^{+}, \mathrm{CD}^{+}, \mathrm{CD}_{1} 9^{+}, \mathrm{CD}_{11} \mathrm{~b}^{+}$, and $\mathrm{CD} 11 \mathrm{c}^{+}$ cells (Figure 1b). IL-10 production increased with the time of incubation of $N$. gonorrhoeae (Figure 1c), and maximal stimulation was achieved at multiplicity of infection (MOI) 10:1; larger numbers of gonococci than MOI 100:1 tended to kill the cultures (data not shown). Prolonged stimulation for up to 14 days did not trigger Th1 or Th2 responses (Figure 1c). Similar levels of cytokine production were obtained when ILN cells were obtained from mice that had been infected in vivo and then further cultured in vitro with $N$. gonorrhoeae; no significant increase in any cytokine occurred (Supplementary Figure S1 online).

To assess whether high level IL-10 production is a common phenomenon following bacterial challenge, we also incubated mouse lymphocytes with another Gram-negative bacterium, Escherichia coli, under the same conditions. E. coli induced much lower levels of IL-10 $(P<0.001)$ but more IL-12p70 $(P<0.001)$ and interferon (IFN)- $\gamma(P<0.01)$, as well as IL-4 and IL-2 (Figure 1a). All these data suggest that infection with different bacteria results in different immune responses characterized by the production of Th lineage-associated cytokines and show that $N$. gonorrhoeae potently elicits the immunosuppressive cytokine, IL-10.

As N. gonorrhoeae induced high levels of IL-10 in the absence of IL-4 and IL-2, we investigated whether it stimulated the generation of $\operatorname{Tr} 1$ cells. Flow cytometry studies revealed that most IL-10-producing $\mathrm{CD} 4{ }^{+} \mathrm{T}$ cells following gonococcal stimulation were $\mathrm{IL}^{-} 4^{-}$and forkhead box $\mathrm{p} 3$ (Foxp3) (Figure 1d), which is consistent with a Tr1 cell classification. By contrast, N. gonorrhoeae did not significantly increase $\mathrm{CD} 4^{+} / \mathrm{Foxp}^{+}$Treg cells (Figure 1d), and $N$. gonorrhoeae-induced Tr1 cells produced very low levels of TGF- $\beta$ (Figure 1e).

To examine the direct effect of $N$. gonorrhoeae on $\mathrm{CD} 4{ }^{+} \mathrm{T}$ cells, similar experiments were performed with purified $\mathrm{CD} 4^{+}$ cells that were stimulated with $N$. gonorrhoeae in the presence of mitomycin C-inactivated APC. Similar results were observed. Supernatants from the cultures at day 4 showed abundant production of IL-10, but not IL-12p70, IFN- $\gamma$, IL-4, or IL-2 (Figure 1f). By contrast, $\mathrm{CD} 4^{+}$cells stimulated with $N$. gonorrhoeae in the absence of APC or OT-II CD4 ${ }^{+}$cells stimulated with $N$. gonorrhoeae plus APC produced 

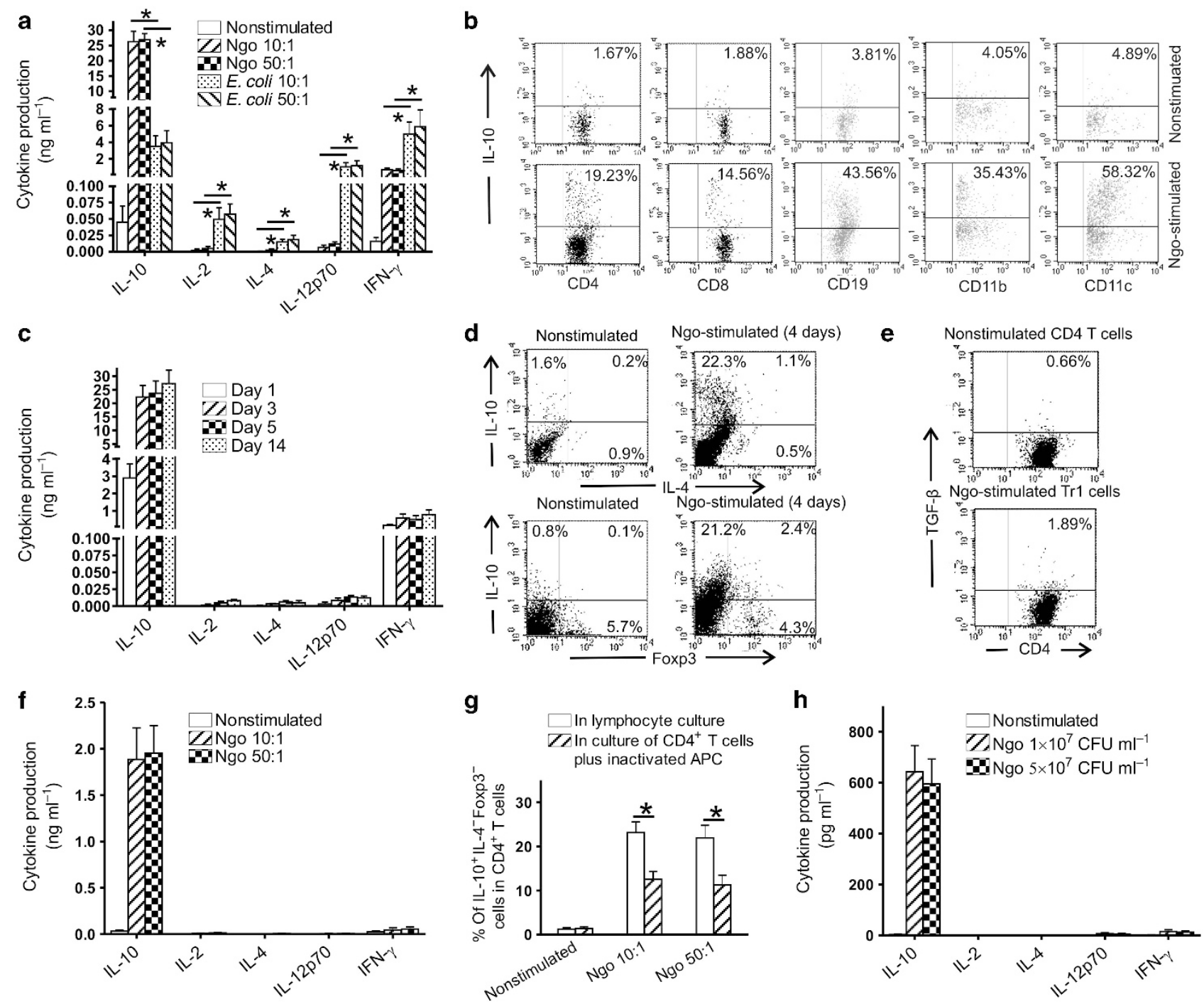

Figure 1 Neisseria gonorrhoeae (Ngo) induces abundant production of interleukin (IL)-10 and type 1 regulatory T (Tr1) cells in BALB/c mouse iliac lymph node (ILN) cells, CD4 ${ }^{+} \mathrm{T}$ cells, and genital tract explants. (a) Cytokine secretion by ILN cells stimulated with $N$. gonorrhoeae or with Escherichia coli (multiplicity of infection (MOI) 10:1 or 50:1). After 4 days of culture, supernatants were collected and the cytokine levels were measured by enzyme-linked immunosorbent assay. (b) Intracellular staining for IL-10 in different immune cell types in ILN cell cultures after stimulation with N. gonorrhoeae (MOI 10:1) for 4 days. Cells were recovered, stained for surface markers and intracellular IL-10, and analyzed by flow cytometry. (c) Time course of cytokine production by ILN cells stimulated with $N$. gonorrhoeae (MOI 10:1). (d) Intracellular staining for IL-10, IL-4, and forkhead box p3 (Foxp3) in CD4 ${ }^{+}$T cells after stimulation with $N$. gonorrhoeae (MOI 10:1) for 4 days. The expression levels of cytokines and Foxp3 were analyzed by flow cytometry with gating on live CD4 ${ }^{+}$T cells. (e) Intracellular staining for transforming growth factor- $\beta$ (TGF- $\beta$ ) in gated CD4 ${ }^{+} / \mathrm{IL}-10^{+} /$ IL-4-/Foxp3 ${ }^{-}$Tr1 cells after stimulation with $N$. gonorrhoeae (MOI 10:1) for 4 days. (f) Cytokine secretion by purified CD4 ${ }^{+} \mathrm{T}^{-}$cells stimulated with N. gonorrhoeae (MOI 10:1 or 50:1) in the presence of mitomycin C-inactivated antigen-presenting cells (APC) for 4 days. (g) Comparison of the percentages of induced Tr1 cells in CD4 ${ }^{+} \mathrm{T}$ cells between ILN cell cultures and purified CD4 ${ }^{+} \mathrm{T}$-cell cultures stimulated with $\mathrm{N}$. gonorrhoeae (MOI 10:1 or 50:1) for 4 days. (h) Cytokine secretion by genital tract explants stimulated in vitro with $N$. gonorrhoeae $\left(1 \times 10^{7}\right.$ or $5 \times 10^{7}$ colony-forming units (CFUs) $\mathrm{ml}^{-1}$ ) for 4 days. All data shown are representative of four independent experiments. ${ }^{*} P<0.01 \mathrm{~N}$. gonorrhoeae- vs. E coli-stimulated (a) or purified CD4 T cells vs. lymphocyte culture (g). IFN, interferon.

significantly less IL-10 (Supplementary Figure S2 online). N. gonorrhoeae could also induce $\mathrm{CD} 4^{+} / \mathrm{IL}-10^{+} / \mathrm{IL}-4^{-} / \mathrm{Foxp}^{-}$ $\mathrm{Tr} 1$ cells in purified $\mathrm{CD} 4^{+} \mathrm{T}$-cell cultures but to a lesser extent (Figure 1g), which suggests that IL-10 from other immune cell types contributes to the process.

To determine the production of cytokines in the mouse genital tract, vaginal explants were cultured with or without $N$. gonorrhoeae. Consistent with the above findings in lymphocyte cultures, IL-10 could be easily detected in the supernatants of vaginal explants stimulated with
$N$. gonorrhoeae, whereas the production of other cytokines, including IL-12p70, IFN- $\gamma$, and IL-4, was minimal (Figure 1h).

\section{N. gonorrhoeae induces IL-10 and Tr1 cells through multiple mechanisms}

Our previous studies have demonstrated that gonococcal Opa proteins contribute to the induction of TGF- $\beta$ and inhibition of Th1/Th2 responses by $N$. gonorrhoeae. ${ }^{15}$ In vivo, Opa proteins have been shown to promote persistence of $N$. gonorrhoeae in the lower genital tract of infected mice. ${ }^{24}$ We therefore 
investigated whether Opa proteins were involved in the elicitation of IL-10 and Tr1 cells in mouse lymphocytes by N. gonorrhoeae, using a mutant of N. gonorrhoeae (FA1090) in which all opa genes have been deleted. Previous colony dot-blot assays have shown that wild-type $N$. gonorrhoeae FA1090 expresses Opa A, B/D/G, and E/K, whereas the Opa-deficient mutant does not express any detectable Opa proteins. ${ }^{15} \mathrm{We}$ cultured ILN cells or purified CD4 ${ }^{+} \mathrm{T}$ cells plus mitomycin C-inactivated APC with Opa-deficient or wild-type N. gonorrhoeae. In both whole lymphocyte and purified $\mathrm{CD} 4^{+} \mathrm{T}$-cell cultures, Opa-deficient $N$. gonorrhoeae induced significantly less IL-10 $(P<0.05)$ and Tr1 cells $(P<0.05)$ in comparison to the wild-type strain (Figure 2a,b). However, the effect is modest, as in the absence of Opa expression N. gonorrhoeae still stimulated considerable levels of IL-10 and numbers of Tr1 cells, suggesting that other gonococcal factors and mechanisms also have an important role in this process.

Gonococcal lipooligosaccharide (LOS) is a critical virulence factor for $N$. gonorrhoeae and is a major inducer of the inflammatory cytokine response to the organism. ${ }^{25,26}$ We next determined whether generation of IL-10 and Tr1 cells was also dependent on LOS-TLR4 interactions. ILN lymphocytes or purified $\mathrm{CD}^{+}{ }^{+} \mathrm{T}$ cells plus inactivated APC from TLR4normal $\mathrm{C} 3 \mathrm{H} / \mathrm{FeJ}$ or TLR4-deficient $\mathrm{C} 3 \mathrm{H} / \mathrm{HeJ}$ mice were stimulated with $N$. gonorrhoeae. Lack of TLR4 resulted in decreased IL-10 $(P<0.001)$ and $\operatorname{Tr} 1$ cell $(P<0.001)$ production in lymph node cell cultures (Figure 2c,d) but did not significantly affect $N$. gonorrhoeae-induced IL-10 and Tr1 cell production in purified $\mathrm{CD} 4{ }^{+} \mathrm{T}$ cells (Figure $2 \mathbf{c}, \mathbf{d}$ ), possibly because the level of TLR 4 expression on $\mathrm{CD}^{+}$cells is relatively low. Other cells in the ILN, such as B cells, may contribute significant amounts of IL-10 production.

\section{IL-10 and Tr1 cells are involved in the suppression of Th1/ Th2 immune responses by $\boldsymbol{N}$. gonorrhoeae}

We have previously demonstrated that $N$. gonorrhoeae selectively suppressed the development of Th1 and Th2 cells. ${ }^{15}$ As $N$. gonorrhoeae can induce abundant production of IL-10 and $\operatorname{Tr} 1$ cells, we determined whether these factors are critically involved in the inhibitory effect of $N$. gonorrhoeae on Th1 and
Th2 responses. Purified mouse $\mathrm{CD} 4{ }^{+} \mathrm{T}$ cells were stimulated with anti-CD3 antibody under Th1-, Th2-, or Th17-polarizing conditions in the additional presence or absence of $N$. gonorrhoeae. IL-10 and Tr1 cell activities in the cultures were blocked by IL-10-neutralizing antibody at a dose determined to be effective without inducing toxicity. Flow cytometric analyses demonstrated that the addition of IL-10-neutralizing antibody significantly reversed $N$. gonorrhoeae-mediated suppression of Th1 and Th2 cell proliferation by $58.9 \pm 7.2 \%(P<0.01)$ and $37.6 \pm 5.3 \%(P<0.05)$, respectively (Figure 3a). Likewise, suppression of IFN- $\gamma$ production in Th1 cells and of IL-5 production in Th2 cells were significantly reversed by IL-10-neutralizing antibody by $62.8 \pm 6.5 \%(P<0.001)$ and $43.5 \pm 5.7 \%(P<0.05)$, respectively (Figure $3 \mathbf{b})$. Similar results were observed when Th1 or Th2 cells from IL-10-knockout $\left(\mathrm{IL}-10^{\mathrm{KO}}\right.$ ) vs. wild-type mice were cultured with $N$. gonorrhoeae (data not shown). All these results indicate that IL-10 and Tr1 cells have an important role in the inhibitory activity of $N$. gonorrhoeae against Th1/Th2-mediated adaptive immunity.

We next compared the effect of IL-10/Tr1 cells and TGF- $\beta$ blockade on the suppression of Th1 and Th2 responses by $N$. gonorrhoeae. Both flow cytometry and enzyme-linked immunosorbent assay (ELISA) showed that anti-IL-10 antibody reversed N. gonorrhoeae-mediated inhibition of Th1 cell development more effectively than anti-TGF- $\beta$ antibody (Figure 3a,b). By contrast, anti-TGF- $\beta$ antibody reversed the inhibition of Th2 cell development to a greater extent (Figure 3a,b). Inhibition of IL-10/Tr1 cells in combination with inhibition of TGF- $\beta$ had an additive effect in reversing the suppression of Th1 and Th2 immunity by $N$. gonorrhoeae $(P<0.05$; Figure $3 a)$. However, in contrast to anti-TGF- $\beta$, antiIL-10 antibody did not block Th17 cell development (Figure 3a,b).

\section{Blockade of IL-10 and Tr1 cell activity enhances Th1/Th2 as well as Th17 immune responses to $\boldsymbol{N}$. gonorrhoeae}

IL-10 and Tr1 cells strongly inhibit Th1 and Th2 immunity. Unlike TGF- $\beta$, IL-10 is not involved in the generation of Th17 cells, and increasing evidence indicates that IL-10 and Tr1 cells also downregulate Th17 immunity. ${ }^{16,19}$ We therefore
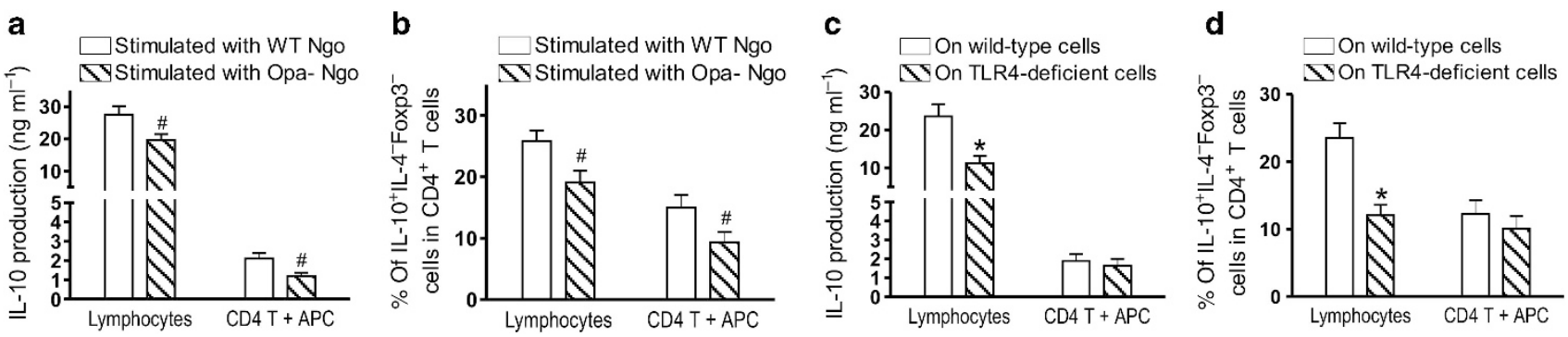

Figure 2 Induction of interleukin (IL)-10 and type 1 regulatory T (Tr1) cells in lymphocytes by Neisseria gonorrhoeae (Ngo) is partially dependent on both opacity (Opa) proteins and lipooligosaccharide-Toll-like receptor 4 (LOS-TLR4) interactions. (a) IL-10 secretion by iliac lymph node (ILN) cells or purified CD4 ${ }^{+} \mathrm{T}$ cells stimulated with wild-type (WT) vs. Opa ${ }^{-} N$. gonorrhoeae (multiplicity of infection (MOI) 10:1) for 4 days. (b) The percentages of induced Tr1 cells in $\mathrm{CD}^{+}{ }^{+} \mathrm{T}$ cells in cultures of ILN cells or purified CD4 ${ }^{+} \mathrm{T}_{\text {cells stimulated with WT vs. Opa }}^{-} \mathrm{N}$. gonorrhoeae (MOI 10:1) for 4 days. (c) IL-10 secretion and (d) Tr1 cell production by WT (C3H/FeJ) vs. TLR4-deficient (C3H/HeJ) ILN or purified CD4 ${ }^{+}$T-cell cultures stimulated with N. gonorrhoeae FA1090 (MOI 10:1) for 4 days. Results from one representative out of four independent experiments are shown. ${ }^{\#} P<0.05$; ${ }^{\star} P<0.01 \mathrm{Opa}^{-}$vs. WT $N$. gonorrhoeae or TLR4-deficient vs. WT cells. APC, antigen-presenting cells; Foxp3, forkhead box p3. 
hypothesized that blockade of IL-10 and Tr1 cell activity would enhance both Th1/Th2 and Th17 immune responses to $N$. gonorrhoeae. We then cultured ILN cells with gonococci in the presence of IL-10-neutralizing antibody or control IgG. Inhibition of IL-10 with a neutralizing antibody dramatically increased $N$. gonorrhoeae-induced IFN- $\gamma$ production $(P<0.001)$ and triggered the production of IL-12p70 and IL-4 $(P<0.001$; Figure 3c). Anti-IL-10 antibody also increased IL-17 production $(P<0.05)$ by lymphocytes stimulated with $N$. gonorrhoeae. The results were supported by reverse transcriptase-PCR (RT-PCR) studies of ROR $\gamma \mathrm{t}$ (retinoic acid-related orphan receptor $\gamma \mathrm{t}$ ), T-bet, and GATA3 expression in N. gonorrhoeae-stimulated $\mathrm{CD} 4{ }^{+} \mathrm{T}$ cells treated with anti-IL-10 antibody in comparison to control IgG (Figure 3d). Similar effects of IL-10 blockade were observed in vaginal explant cultures stimulated with $N$. gonorrhoeae, but the levels of IL-12p70, IFN- $\gamma$, and IL-4 production that anti-IL-10 antibody could support in vaginal cultures were relatively low (Supplementary Figure S3 online).

At present, it remains controversial whether IL-10 and TGF$\beta$ enhance the expression of each other. Our results revealed that neutralization of IL-10 and TGF- $\beta$ in the cultures did not significantly decrease the production of each other (Figure 3c), which suggests that IL- 10 and Tr1 cells or TGF- $\beta$ represent two distinct mechanisms involved in the suppressive effect of $N$. gonorrhoeae on Th1/Th2 immunity. Consistent with above results, both ELISA and RT-PCR analyses indicated that blockade of both IL-10 and TGF- $\beta$ has an additive effect in promoting Th1 and Th2 immune responses against N. gonorrhoeae (Figure 3c,d).

\section{IL-10 and Tr1 cell production in murine vaginal gonococcal infection}

To determine whether N. gonorrhoeae induces IL-10 and Tr1 cells in vivo, we examined the local immune response in a mouse model of genital tract gonococcal infection. ILN and vaginas were removed from each of the five mice per group at 1 , $3,5,7$, and 14 days after inoculation with $N$. gonorrhoeae or vehicle only. Single-cell suspensions were prepared from each specimen for evaluation by flow cytometry to detect the expression of IL-10, IL-4, and Foxp3. Starting on day 3 after inoculation, IL-10 production was observed in various immune cells present in the ILN, including $\mathrm{CD} 4^{+}, \mathrm{CD} 8^{+}, \mathrm{CD} 19^{+}$, $\mathrm{CD}_{11} \mathrm{~b}^{+}$, and $\mathrm{CD} 11 \mathrm{c}^{+}$cells (Figure 4a). IL-10 production peaked at day 5 and continued for the duration of infection. At day $5,11.6-20.2 \%$ of the isolated immune cell types in the lymph nodes of infected mice were IL- $10^{+}$, significantly higher than the levels in sham-infected controls (Figure 4a). Almost all IL- $10^{+} / \mathrm{CD} 4^{+} \mathrm{T}$ cells were IL- $4^{-}$(Figure $4 \mathbf{b}$ ) and few were Foxp $^{+}$(Figure 4c), a phenotype indicative of $\operatorname{Tr} 1$ cells. Similar results were observed in flow cytometric analysis of the infiltrating leukocytes in the vaginas (data not shown). The findings were further supported by quantitative RT-PCR analysis of mouse vaginal mRNA, which demonstrated that IL10 was elevated by a factor of $10.4 \pm 3.8$ in $N$. gonorrhoeaeinfected mice relative to control mice $(P<0.001)$. All these data indicated that $N$. gonorrhoeae elicits abundant production of IL-10 and Tr1 cells in vivo in a murine model of vaginal gonococcal infection.

ELISA analysis of vaginal wash, saliva, and serum of $N$. gonorrhoeae-infected and sham-infected mice showed a peak level of IL-10 $\left(\sim 60-90 \mathrm{pg} \mathrm{ml}^{-1}\right)$ in vaginal washes from infected mice at days $3-5$ after inoculation. However, IL-10 was not detected in saliva or serum samples of any mouse at any time point.

\section{Blockade of IL-10 and Tr1 cell activity enhances both Th1/ Th2-mediated adaptive immune responses and Th17- governed innate responses in vaginal gonococcal infection} Given the strong induction of IL-10 and Tr1 cells by $N$. gonorrhoeae and their known suppressive effect on Th1/Th2 immunity, we hypothesized that blockade of IL-10 and Tr1 cells would elicit host Th1 and Th2 adaptive responses to gonococcal
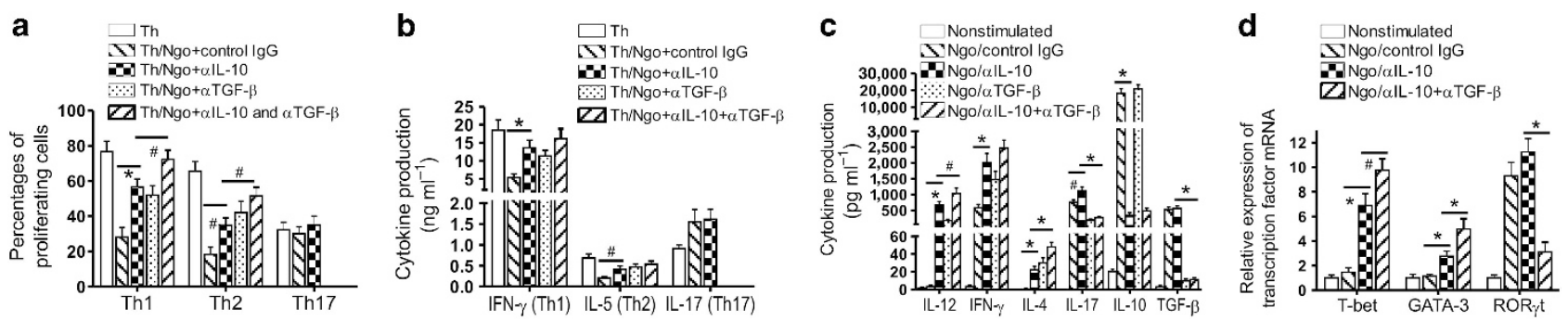

Figure 3 Induction of interleukin (IL)-10 and type 1 regulatory T cells contributes to the inhibitory effect of Neisseria gonorrhoeae (Ngo) on development of BALB/c mouse T helper type 1 (Th1) and Th2 cells, and blockade of these mechanisms promotes Th1 and Th2 as well as Th17 responses. (a) Suppression of cell proliferation in Th1- and Th2-polarized CD4 ${ }^{+}$cell cultures by N. gonorrhoeae (multiplicity of infection (MOI) $50: 1$ ) was reversed by treatment with anti-IL-10 $\left(40 \mu \mathrm{g} \mathrm{ml}^{-1}\right)$ or/and transforming growth factor- $\beta$ (TGF- $\beta$ ) antibody $\left(40 \mu \mathrm{g} \mathrm{ml}^{-1}\right)$. The sequential loss of CFSE (carboxymethyl fluorescein succinimide ester) fluorescence was used to measure cell proliferation. (b) Suppression of interferon (IFN)- $\gamma$ and IL-5 production in Th1- and Th2-polarized CD4 ${ }^{+}$cell cultures, respectively, by $N$. gonorrhoeae (MOI 50:1) was reversed by treatment with anti-IL-10 or/and TGF- $\beta$ antibody. (c) Effect of anti-IL-10 antibody alone or in combination with anti-TGF- $\beta$ antibody on cytokine response of iliac lymph node (ILN) cells stimulated with N. gonorrhoeae (MOI 10:1) for 4 days. (d) Effect of anti-IL-10 antibody alone or in combination with anti-TGF- $\beta$ antibody on expression of T-bet, GATA3, and ROR $\gamma \mathrm{t}$ (retinoic acid-related orphan receptor $\gamma \mathrm{t}$ ) in CD4 ${ }^{+} \mathrm{T}$ cells MACS (magnetic-activated cell sorter)-purified from ILN cultures stimulated with N. gonorrhoeae (MOI 10:1) for 4 days. Transcription factor gene expression levels detected by reverse transcriptase-PCR were normalized relative to expression of $\beta$-actin and set at 1.0 for unstimulated cells. All data shown are representative of four independent experiments. ${ }^{\#} P<0.05$;

${ }^{*} P<0.01$ anti-IL-10 vs. control immunoglobulin G (IgG) treatments or anti-IL-10 plus anti-TGF- $\beta$ vs. anti-IL-10 treatments. 

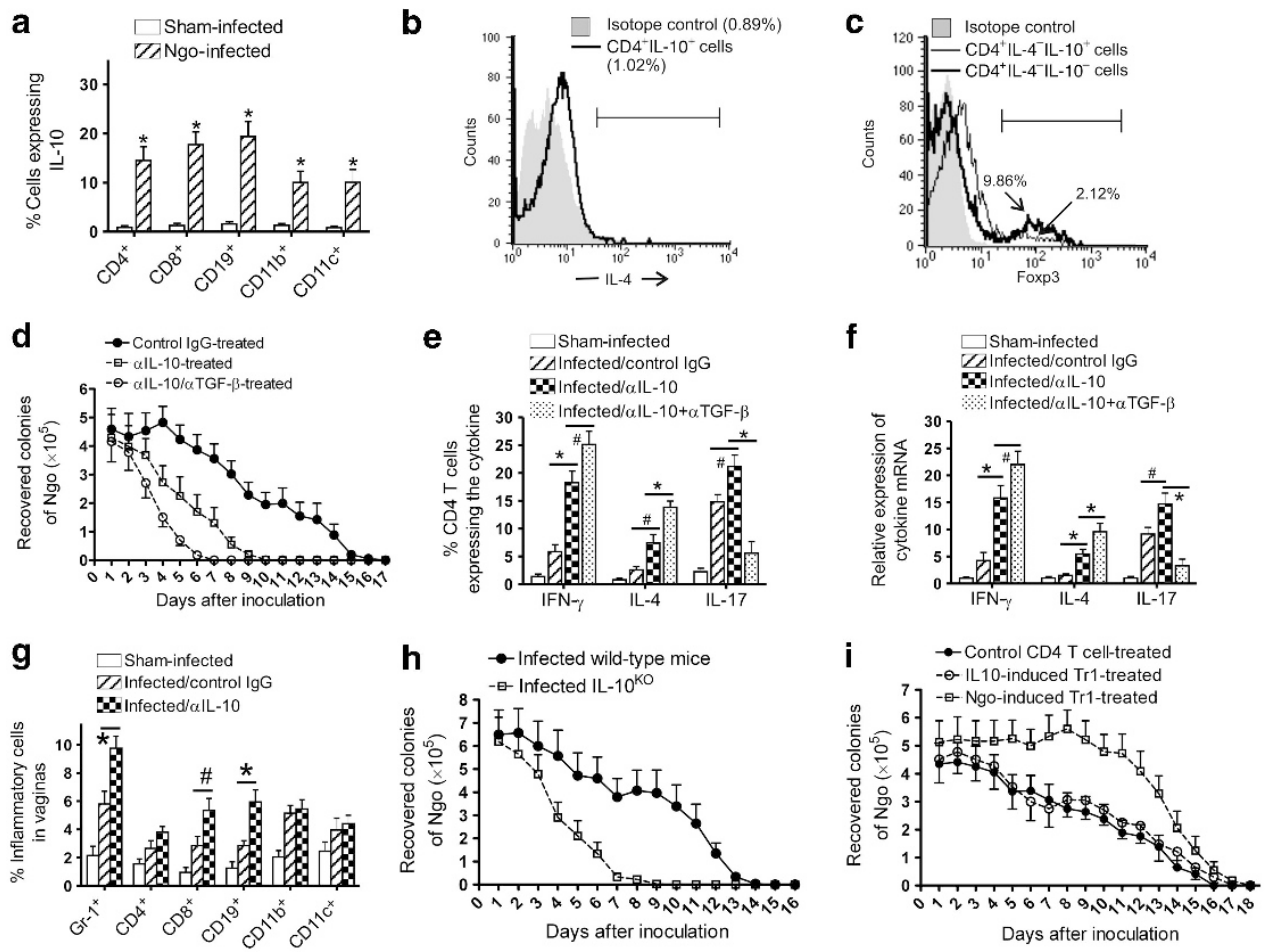

Figure 4 Induction and role of interleukin (IL)-10 and type 1 regulatory T (Tr1) cells in mice during vaginal gonococcal infection. (a) IL-10 production by iliac lymph node (ILN) cells isolated from Neisseria gonorrhoeae ( $\mathrm{Ngo}$ )-infected or sham-infected mice 5 days after inoculation and analyzed by flow cytometry; $n=8$ mice per group. (b) Intracellular staining for IL-4 in CD4 ${ }^{+} / \mathrm{IL}-10^{+}$T cells isolated from draining lymph nodes of $N$. gonorrhoeae-infected mice at day 5. The horizontal line shows the gate of IL-4 positivity set by reference to the isotype control. (c) Intracellular staining for forkhead box $\mathrm{p} 3$ (Foxp3) in CD4 ${ }^{+} / \mathrm{IL}-4^{-} / \mathrm{IL}-10^{+}$or CD4 $4^{+} / \mathrm{IL}-4^{-} / \mathrm{IL}-10^{-} \mathrm{T}$ cells isolated from ILN of $N$. gonorrhoeae-infected mice at day 5 . (d) Time course of $N$. gonorrhoeae infection in anti-IL-10 antibody-treated, anti-IL-10 plus anti-TGF- $\beta$ (anti-transforming growth factor- $\beta$ ) antibody-treated, and control immunoglobulin G (IgG)-treated mice; $n=8$ mice per group. (e) Cytokine expression in isolated ILN cells from sham-infected or infected mice treated with anti-IL-10 antibody, anti-IL-10 plus anti-TGF- $\beta$ antibody, or control IgG; $n=8$ mice per group. Expression of interferon (IFN)- $\gamma$, IL-4, and IL-17 in CD4 ${ }^{+}$T cells at day 5 was analyzed by flow cytometry. (f) Reverse transcriptase-PCR (RT-PCR) analysis of IFN- $\gamma$, IL-4, and IL-17 mRNA levels in vaginal tissue harvested at day 3 from sham-infected or from infected mice treated with anti-IL-10 antibody, anti-IL-10 plus anti-TGF- $\beta$ antibody, or control IgG; $n=7$ mice per group. Cytokine gene expression levels detected by RT-PCR were normalized relative to expression of $\beta$-actin and set at 1.0 for unstimulated cells. (g) Phenotypic profile of vaginal cells isolated on day 5 from sham-infected or infected mice treated with anti-IL-10 antibody or control IgG; $n=7$ mice per group. (h) Time course of $N$. gonorrhoeae infection in wild-type vs. IL-10-knockout (IL-10 $\mathrm{KO}$ ) C57BL/6 mice; $n=8$ mice per group. (i) Time course of $N$. gonorrhoeae infection mice receiving adoptively transferred IL-10-induced Tr1 cells, gonococcus-induced Tr1 cells, or control CD4 ${ }^{+}$T cells; $n=8$ mice per group. Results from one representative out of three independent experiments are shown. ${ }^{\#} P<0.05 ;{ }^{*} P<0.01$ $N$. gonorrhoeae- vs. sham-infected groups, anti-IL-10 vs. control IgG treatments, or anti-IL-10 plus anti-TGF- $\beta$ vs. anti-IL-10 treatments.

infection and enhance bacterial clearance. To test this, groups of female $\mathrm{BALB} / \mathrm{c}$ mice were vaginally infected with $N$. gonorrhoeae, and the bacterial burden was monitored daily by vaginal swab culture. The mice were injected with $300 \mu \mathrm{g}$ of IL-10-neutralizing antibody or control IgG on days -1 and 0 and every 2 days thereafter. Preliminary experiments showed this dose to be optimal without generating side-effects. The duration of gonococcal infection in control IgG-treated mice was $\sim 15$ days (Figure $4 d$ ). Anti-IL-10 treatment significantly reduced the recoverable gonococcal load starting from day 4 , and led to faster clearance of the infection $\left(F_{1,238}=69.20\right.$; $P<0.001$; Figure 4d). Flow cytometric analysis demonstrated that inhibition of IL-10 and Tr1 cells resulted in enhanced Th1 and Th2 immune responses, indicated by significantly increased numbers of IFN- $\gamma^{+} / \mathrm{CD}^{+} \quad(P<0.01)$ and $\mathrm{IL}-4^{+} / \mathrm{CD} 4^{+} \mathrm{T}$ cells $(P<0.05)$ in the ILN from treated mice in comparison to those from control mice (Figure 4e). In contrast to the effect of anti-TGF- $\beta$ observed in our previous study, ${ }^{14,15}$ anti-IL-10 did not hinder the Th17 response; instead it elevated the number of $\mathrm{IL}-17^{+} / \mathrm{CD} 4^{+} \mathrm{T}$ cells $(P<0.05)$ in ILN (Figure 4e). Similar results were observed in vaginas of the same mice. RT-PCR analyses showed that IFN- $\gamma(P<0.001)$, IL-4 $(P<0.01)$, and IL-17 $(P<0.05)$ mRNA expressions were all enhanced in the infected mice following anti-IL-10 antibody treatment (Figure 4f). In addition, anti-IL-10 increased Gr- ${ }^{+}$ neutrophil $(P<0.01), \mathrm{CD}^{+}(P<0.05)$, and $\mathrm{CD} 19^{+}$cell $(P<0.01)$ influx into the genital tract (Figure $4 \mathbf{g}$ ). Consistent with the in vitro experiments, these in vivo findings revealed that inhibition of IL-10/Tr1 cell and TGF$\beta$ activities had an additive effect in overcoming the suppression of Th1 and Th2 immunity by gonococci (Figure 4e,f). Treatment of infected mice with both anti-IL-10 and antiTGF- $\beta$ further accelerated clearance of $N$. gonorrhoeae $\left(F_{1,238}=13.31 ; P<0.01\right.$; Figure 4d $)$.

To confirm the importance of IL-10 and Tr1 cells in the response to vaginal infection with $N$. gonorrhoeae, we repeated 
the experiment using $\mathrm{IL}-10^{\mathrm{KO}}$ (C57BL/6 background) mice, which lack the capability to generate IL-10 and Tr1 cells. Gonococcal infection in wild-type C57BL/6 mice lasted about 2 weeks, whereas IL-10 ${ }^{\mathrm{KO}}$ mice started to show diminished gonococcal recovery from day 3 and had cleared the infection by day $7\left(F_{1,224}=61.74 ; P<0.001\right.$; Figure $\left.4 \mathbf{h}\right)$.

As IL-10 was produced by a variety of immune cell types in addition to $\operatorname{Tr} 1$ cells, we then investigated the specific role of $\operatorname{Tr} 1$ cells in an adoptive transfer experiment. IL-10- or $N$. gonorrhoeae-induced $\operatorname{Tr} 1$ cells were generated by prolonged culture of $\mathrm{CD}^{+}$cells from $\mathrm{BALB} / \mathrm{c}$ mice in the presence of IL-10 or N. gonorrhoeae, as described in Methods. Groups of BALB/c mice infected with $N$. gonorrhoeae were injected intravenously on days 0 and 3 with $1 \times 10^{6} \operatorname{Tr} 1$ or control CD4 ${ }^{+} \mathrm{T}$ cells. Transfer of $N$. gonorrhoeae-induced $\left(F_{1,252}=64.03 ; P<0.01\right)$, but not IL-10-induced $\left(F_{1,252}=1.06\right.$; $P=0.31)$, Tr1 cells elevated the recoverable gonococcal burdens in the treated mice from day 5 through 13 after inoculation (Figure 4i). However, the transfer of exogenous Tr1 cells did not prolong the duration of infection, possibly because large numbers of these cells were induced endogenously in the animals by infection with $N$. gonorrhoeae.

\section{Treatment with anti-IL-10 antibody protects mice against secondary $\boldsymbol{N}$. gonorrhoeae infection}

As inhibition of IL-10 and Tr1 cells elicited the development of Th1/Th2 adaptive responses to primary gonococcal infection, we then assessed whether the treatment resulted in the generation of immune memory and protective immunity. Reinfection studies were performed in which groups of mice treated with anti-IL-10 or control IgG during primary infection with $N$. gonorrhoeae were allowed to recover and then treated with ceftriaxone on day 15 to ensure complete clearance of the infection. An additional group of sham-infected mice treated with anti-IL-10 was used to evaluate the possible persistent effect of anti-IL-10 antibody in the absence of infection. Fiveto-six weeks later, all mice were inoculated with $N$. gonorrhoeae of the same strain without any further treatment. Consistent with previous studies, ${ }^{14}$ the duration and bacterial burden of secondary infection in previously control IgG-treated mice were the same as for the primary infection of age-matched naive mice $\left(F_{1,294}=3.25 ; P=0.09\right.$; Figure $\left.5 \mathbf{a}\right)$. By contrast, anti-IL-10 treatment during primary $N$. gonorrhoeae infection protected the mice against secondary infection. Reinfected mice that had been treated with anti-IL-10 antibody during the primary infection recovered significantly faster than controls $\left(F_{1,294}=99.13 ; P<0.001\right.$; Figure 5a). Interestingly, previous anti-IL-10 treatment of sham-infected mice did not induce protection against subsequent infection $\left(F_{1,294}=0.44 ; P=0.51\right.$; Figure 5a). We also found that treatment with anti-IL-10 combined with anti-TGF- $\beta$ during primary gonococcal infection protected the mice more effectively than treatment with anti-IL-10 alone $\left(F_{1,294}=10.68 ; P<0.05\right.$; Figure 5a).

Flow cytometric and RT-PCR studies of ILN and vaginas taken on day 5 indicated that the protective effect of previous anti-IL-10 treatment on secondary gonococcal infection was also associated with enhanced Th1 (IFN- $\gamma ; P<0.001)$ and Th2 (IL-4; $P<0.01$ ) responses (Figure 5b,c). However, Th17 response (IL-17) in the reinfected mice with previous antiIL-10 treatment was similar to those receiving control IgG treatment.

We then tested whether inhibition of IL-10 and Tr1 cells during N. gonorrhoeae infection led to the production of anti-gonococcal antibodies. IgM antibodies showed low levels and little difference between experimental groups (data not shown). No salivary gonococcus-specific antibody was detected in any group of mice (data not shown). Without anti-IL-10 treatment, neither primary nor secondary $N$. gonorrhoeae infection of mice induced the production of significant gonococcus-specific IgA or IgG antibody responses in either serum or vaginal wash (Figure 6a-d). There was also no significant increase in specific IgA or IgG antibody responses in serum or vaginal wash to primary infection with anti-IL-10 treatment (Figure 6a,b). However, there was a specific antibody response in anti-IL-10-treated mice after they were re-exposed to N. gonorrhoeae. Gonococcus-specific IgG antibodies in

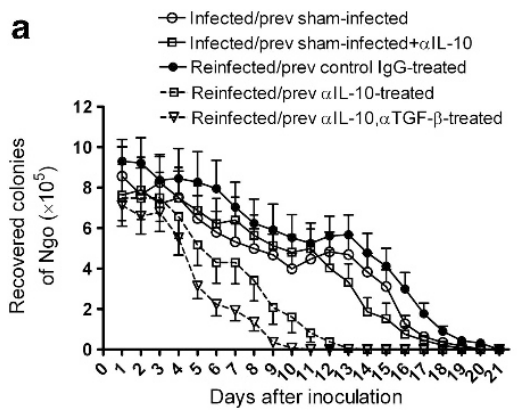

b

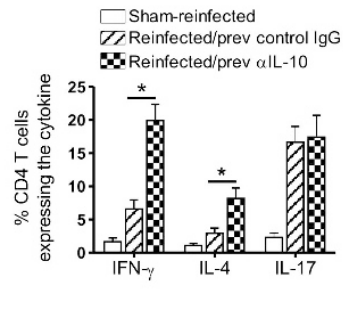

C

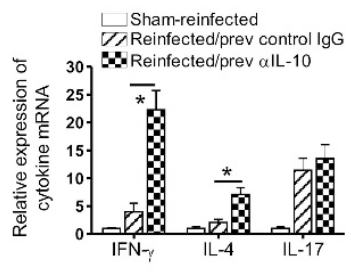

Figure 5 Effect of treatment with anti-interleukin (IL)-10 antibody alone or with anti-TGF- $\beta$ (anti-transforming growth factor- $\beta$ ) antibody during primary infection on secondary gonococcal infection in BALB/c mice. (a) Time course of secondary infection in mice treated with anti-IL-10 antibody, anti-IL-10 plus anti-TGF- $\beta$, or control immunoglobulin G (IgG) during primary infection or in previously sham-infected mice with or without anti-IL-10 treatment; $n=8$ mice per group. (b) Flow cytometric analysis of cytokine expression in iliac lymph node CD4 ${ }^{+} \mathrm{T}$ cells isolated at day 5 from previously sham-infected or from reinfected mice treated with anti-IL-10 antibody or control IgG during primary infection; $n=8$ mice per group. (c) Reverse transcriptase-PCR (RT-PCR) analysis of interferon (IFN)- $\gamma$, IL-4, and IL-17 mRNA levels in vaginas harvested at day 3 from previously sham-infected or from reinfected mice treated with anti-IL-10 antibody or control IgG during primary infection; $n=7$ mice per group. Cytokine gene expression levels detected by RT-PCR were normalized relative to expression of $\beta$-actin and set at 1.0 for unstimulated cells. All data shown are representative of three independent experiments. ${ }^{*} P<0.01$ anti-IL-10 vs. control IgG treatments. Ngo, Neisseria gonorrhoeae. 

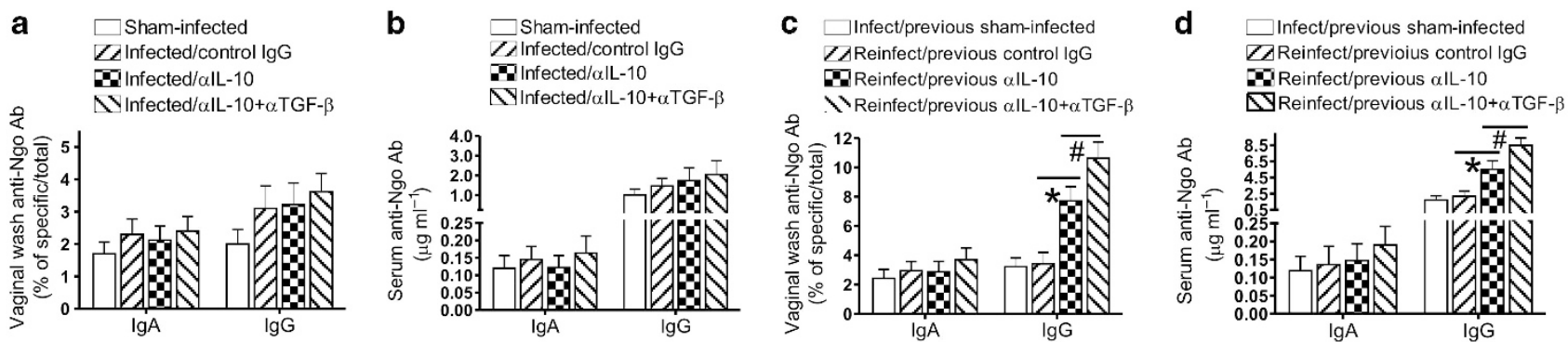

Figure 6 Anti-gonococcal antibody (Ab) responses to primary and secondary infection in anti-interleukin (IL)-10 antibody-treated, anti-IL-10 plus anti-TGF- $\beta$ (anti-transforming growth factor- $\beta$ ) antibody-treated, control immunoglobulin G (IgG)-treated, or sham-infected BALB/c mice. (a) Vaginal IgA and IgG responses to primary infection. (b) Serum IgA and IgG responses to primary infection. (c) Vaginal IgA and IgG responses to secondary infection. (d) Serum IgA and IgG responses to secondary infection. Vaginal washes and sera were collected 15 days after inoculation, and gonococcus-specific and total IgA and IgG were measured by enzyme-linked immunosorbent assay. Data shown are from one experiment using seven mice per group. ${ }^{\#} P<0.05 ;{ }^{*} P<0.01$ anti-IL-10 vs. control IgG treatments or anti-IL-10 plus anti-TGF- $\beta$ vs. anti-IL-10 treatments. Two more replicate experiments yielded essentially similar results and levels of significance. Ngo, Neisseria gonorrhoeae.

vaginal wash and serum of reinfected mice with previous antiIL-10 treatment were significantly higher than those of controls $(P<0.01$; Figure 6c,d $)$. However, anti-IL-10 did not increase the production of specific IgA antibody in either serum or vaginal wash. Furthermore, we found that inhibition of both IL-10 and TGF- $\beta$ during primary $N$. gonorrhoeae infection had an additive effect in enhancing the gonococcus-specific IgG antibody responses in both vaginal wash and serum to subsequent infection $(P<0.05$; Figure $\mathbf{6 c}, \mathbf{d})$.

\section{DISCUSSION}

We have previously shown that vaginal infection of mice with $N$. gonorrhoeae selectively elicits IL-17-dependent innate responses and concomitantly suppresses Th1/Th2-driven adaptive immune responses through mechanisms that depend on TGF- $\beta .^{13-15}$ Administration of anti-TGF- $\beta$ antibody alleviates this suppression and permits the development of Th1 and Th2 responses, as well as the establishment of immune memory and the generation of anti-gonococcal antibodies, thereby accelerating clearance of a primary gonococcal infection and protecting against a subsequent infection. ${ }^{14,15}$

The involvement of TGF- $\beta$ in these effects suggests a role for Treg cells, which have been previously reported in the mouse model of genital gonococcal infection. ${ }^{27}$ However, we noted that $N$. gonorrhoeae did not strongly induce expression of the nuclear transcription factor, Foxp3, that is associated with conventional induced Treg cell function. ${ }^{15}$ We now show that N. gonorrhoeae induced very high levels of IL-10 and Tr1 cells both in cultured lymphoid cells in vitro and in cells recovered from mice vaginally infected with N. gonorrhoeae. As OT-II $\mathrm{CD} 4{ }^{+}$cells stimulated with $\mathrm{N}$. gonorrhoeae or wild-type $\mathrm{CD} 4{ }^{+}$ cells stimulated with $N$. gonorrhoeae in the absence of APC produced significantly less IL-10, it is likely that a significant proportion of $N$. gonorrhoeae-activated IL-10-producing $\mathrm{CD}^{+}$cells are gonococcus-specific. The generation of IL-10 appeared to be a specific effect of N. gonorrhoeae, as culture of cells with another gram-negative bacterium, E. coli, induced much lower levels of IL-10 and much more IFN- $\gamma$ and IL-12. An Opa-deficient mutant of N. gonorrhoeae induced slightly lower levels of IL-10 in mouse cells in vitro, suggesting that other gonococcal components probably have an important role in this process. However, we could not determine whether a similar outcome occurs in vivo because this Opa-deficient mutant appears to have growth defects, which compromise its ability to infect mice. Moreover, it is unclear at present what receptors are engaged by gonococcal Opa proteins, as murine CEACAM receptors (unlike human CEACAMs) do not bind Opa. Unfractionated lymph node cells from TLR4-deficient mice were significantly less able to generate IL-10 in response to stimulation with $N$. gonorrhoeae, implying that gonococcal LOS contributes to this effect, but purified $\mathrm{CD}^{+}{ }^{+} \mathrm{T}$ cells (plus APC) did not replicate this effect, suggesting that LOS-responsive cells were not sufficiently represented in these cultures. Although TLR4 is expressed on myeloid cells, including APC, it is not present at high levels on murine $\mathrm{T}$ cells. We found previously that murine B cells, which are known to express TLR4, contribute to the LOS-stimulated generation of Th17 responses. ${ }^{15}$ Others have found that TLR4-deficient mice infected with N. gonorrhoeae show higher peak bacterial loads and diminished Th17-related cytokine responses, but IL10 was not examined. ${ }^{25}$ However, as TLR4-deficiency impacts on many other aspects of the inflammatory response to infection in addition to IL-10 production, this outcome is not necessarily at variance with our findings.

Although many cell types secreted IL-10 in response to gonococcal stimulation, we found that the IL-10-producing $\mathrm{CD} 4^{+} \mathrm{T}$ cells were mostly $\mathrm{IL}-4^{-} / \mathrm{Foxp}^{-}$, a phenotype characteristic of $\operatorname{Tr} 1$ cells. ${ }^{18}$ This subset of Treg cells is known to suppress Th1, Th2, and also Th17 cells. ${ }^{16,19,28}$ Anti-IL-10 antibody reversed the suppression of Th1 and Th2 cells by $N$. gonorrhoeae without affecting the induction of Th17 cells. AntiTGF- $\beta$ antibody had an additive effect to anti-IL-10 on the reversal of Th1 and Th2 suppression, but it also counteracted the induction of Th17 cells, which require TGF- $\beta$ for their generation.

These findings from in vitro experiments were reflected in the tissues of mice infected with $N$. gonorrhoeae in vivo. Thus lymphoid cells isolated from vaginal tissue and from the 
ILN, which drain the genital tract, of $N$. gonorrhoeae-infected mice showed production of IL-10 by CD $4^{+}$and $\mathrm{CD} 8{ }^{+} \mathrm{T}$ cells, $\mathrm{B}$ cells, as well as both $\mathrm{CD} 11 \mathrm{~b}^{+}$and $\mathrm{CD} 11 \mathrm{c}^{+}$cells, which are taken to be macrophages and dendritic cells, respectively. The selective induction of IL-10 in dendritic cells by $N$. gonorrhoeae has recently been reported. ${ }^{12}$ Furthermore, the CD4 ${ }^{+}$cells that produced IL-10 in vivo were largely $\mathrm{IL}_{-}^{-}{ }^{-}$and Foxp3-, corresponding to the $\operatorname{Tr} 1$ phenotype. Accordingly, treatment of mice with blocking antibody to IL-10 during vaginal gonococcal infection accelerated the clearance of the infection and enhanced the generation of Th1 and Th2 cells without impairing the generation of Th17 cells. Co-administration of anti-TGF- $\beta$ antibody with anti-IL-10 resulted in even faster clearance. In addition, IL-10-deficient mice were significantly more resistant to vaginal gonococcal infection than their wildtype counterparts. This phenotype is similar to that of IL-22knockout mice, which are also resistant to vaginal gonococcal infection. ${ }^{29}$ Beyond noting that IL-22 is structurally related to IL-10, shares the IL-10R2 signaling component in its receptor, and signals through Jak-STAT (janus kinase- signal transducer and activator of transcription) pathways, ${ }^{17}$ we have no satisfactory explanation for the latter observation, as the two cytokines target different cells and have quite different biological effects.

To test directly the involvement of $\operatorname{Tr} 1$ cells in the suppression of immune responses by $N$. gonorrhoeae, we performed an adoptive transfer experiment. ILN cells from $N$. gonorrhoeae-infected mice were cultured for a prolonged period with gonococci of the same strain in the presence of antiCD3 antibodies, which led to the generation of gonococcusspecific Tr1 cells. Adoptive transfer of these cells into congenic mice that were infected with N. gonorrhoeae impaired early reduction of the bacterial load. Non-specific Tr1 cells generated by culture with IL-10 did not have this effect. However, transfer of gonococcus-specific Tr1 cells did not prolong the total course of infection, and we ascribe that to the endogenous generation of $\operatorname{Tr} 1$ cells as a consequence of infection. In these circumstances, it seems likely that the adoptive transfer of additional, exogenously generated $\operatorname{Tr} 1$ cells served to suppress the early response but did not alter the ultimate outcome.

We previously demonstrated that treatment of mice with blocking antibodies to TGF- $\beta$ during primary gonococcal infection leads to the establishment of Th1- and Th2-driven immune memory that can be recalled upon subsequent reinfection with $N$. gonorrhoeae. ${ }^{14}$ As a result, the second infection is cleared more effectively, and anti-gonococcal IgA and IgG antibodies are found in the serum and genital secretions-neither of which outcomes occurs normally in reinfected mice. ${ }^{14,30}$ We found similar development of protective anamnestic immune responses, including antigonococcal IgG antibodies, when mice that were treated with anti-IL-10 antibody during primary infection were challenged a second time but without further treatment with anti-IL-10 antibody. Moreover, treatment with anti-TGF- $\beta$ in addition to anti-IL-10 antibody increased the effect. Similar to blockade of
TGF- $\beta$, which promotes both Th1 and Th2 anamnestic responses, ${ }^{14}$ blockade of IL-10 enhanced Th1 and Th2 responses measured by the expression and production of IFN- $\gamma$ and IL- 4 in the genital tract tissues and draining ILN. However, in contrast to anti-TGF- $\beta$, which facilitates the generation of both IgG and IgA anti-gonococcal antibody responses, ${ }^{14}$ anti-IL-10 treatment enhanced only IgG antibody responses in serum and vaginal secretion, and unlike antiTGF- $\beta$, it did not inhibit the IL-17 response.

From these findings and our previous studies, we conclude that $N$. gonorrhoeae suppresses Th1- and Th2-driven adaptive immune responses against itself by mechanisms that depend upon TGF- $\beta$ and IL-10 and that involve the generation of Tr1 cells, which are known to inhibit Th1 and Th2 cells. This induced, specific immunosuppression can be reversed by blockade of IL-10 and/or TGF- $\beta$, thereby allowing the induction of Th1 and Th2 cells, the establishment of immune memory, the generation of specific anti-gonococcal antibodies, and the development of protective immunity. This has not previously been accomplished in models of genital tract infection with N. gonorrhoeae.

We have proposed that the suppression of Th1- and Th2driven specific immune responses coupled with the selective elicitation of Th17-dependent innate immune responses is an adaptive strategy that enables $N$. gonorrhoeae to colonize its host by proactively directing the pattern of host immune responses in its own favor. ${ }^{31} \mathrm{~N}$. gonorrhoeae appears well able to resist innate defense mechanisms driven by Th17 responses, as it can survive within neutrophils ${ }^{32,33}$ and resist antibacterial proteins such as defensins by means of a multi-drug exporter mechanism. ${ }^{34}$ Under this hypothesis, we propose that Th1/ Th2-driven adaptive immune responses, such as the generation of specific anti-gonococcal antibodies, provide the key additional factor that arms host defenses effectively against $N$. gonorrhoeae. It is unclear, at present, whether Th17-driven responses, such as the neutrophil influx, are essential for clearance of vaginal gonococcal infection in mice. IL-17 receptor A-knockout mice, or normal mice treated with IL17-blocking antibody, show prolonged infection and diminished neutrophil influx into the genital tract. ${ }^{13}$ However, the association of these two effects may be coincidental, because the neutrophil influx varies between different strains of mice regardless of the clearance kinetics. ${ }^{35}$ Moreover, in our studies of murine vaginal gonococcal infection, clearance still occurred in IL-17 receptor A-knockout mice despite the absence of a neutrophil influx. ${ }^{13}$ It is likely that numerous factors are responsible for the elimination of vaginal gonococcal infection in mice, including not only host responses to the infection but also the fact that $N$. gonorrhoeae is an exclusively human pathogen that has become evolutionarily well adapted to its sole natural host and is not ordinarily capable of infecting mice. However, it is possible that these effects are limited to the artificial murine infection, and therefore it is critical to determine the extent to which they apply to the natural human infection. It has already been reported that humans infected with gonorrhea have elevated serum IL-17 and 
IL-23, ${ }^{36}$ but it is not clear whether this is a consequence of infection or a predisposing factor in acquiring the infection.

The preponderance of clinical experience shows that episodes of gonorrhea do not induce effective immunity against re-infection, as gonorrhea can be re-acquired with no apparent reduction in severity or duration of disease. The conventional explanations adduced for this situation include the extraordinary capacity of $N$. gonorrhoeae for antigenic variation involving most of its surface antigens, ${ }^{5}$ coupled with numerous mechanisms for inhibiting complement activation and resisting cytolysis. ${ }^{10}$ It is also becoming clear that $N$. gonorrhoeae can survive phagocytosis by neutrophils, the predominant cells found in the exudates that are characteristic of symptomatic gonorrhea, by interfering with both oxygendependent and oxygen-independent intracellular killing. ${ }^{32,33}$ Although all of these mechanisms undoubtedly contribute to the success of $N$. gonorrhoeae as a pathogen, we propose that it also suppresses specific adaptive immune responses, including antibody development, that might be capable of eliminating it. Our findings reported here and previously support this concept and indicate some of the mechanisms whereby this is achieved.

Our findings are complemented by a recent report that $N$. gonorrhoeae interacts with dendritic cells to induce the production of not only IL-10 but also programmed death ligand $1 .^{12}$ As a result, such gonococcus-exposed dendritic cells suppress antigen-induced proliferation of $\mathrm{CD} 4{ }^{+} \mathrm{T}$ cells. It had previously been shown that $N$. gonorrhoeae can inhibit the proliferation of human $\mathrm{CD} 4{ }^{+} \mathrm{T}$ cells through the binding of gonococcal Opa proteins to it receptor, CEACAM-1, which is expressed on these cells. ${ }^{11}$ A similar effect on human B cells was also reported. ${ }^{37}$ Thus it appears that $N$. gonorrhoeae can interact with host immune cells at multiple levels, i.e., with APC, Th cells, and B cells, as well as Tr1 cells, to suppress the development of specific immune responses. We propose that if specific immune responses to $N$. gonorrhoeae were allowed to proceed in the normal manner, then the effector products of such immune activation, particularly including anti-gonococcal antibodies, might be able to eliminate the infection. Moreover, the concomitant establishment of immune memory might provide protection against re-infection, assuming a sufficient degree of antigenic similarity between the original and new infecting strains. Possibly this occurs eventually in natural human infections if they are allowed to proceed untreated, and anecdotal evidence from the pre-antibiotic era suggests that gonorrhea is ultimately self-limiting. ${ }^{38}$ However, it is completely unethical to test such speculations directly in humans. Instead, our evidence from experimental murine infections indicates that it is possible to immunologically reverse the natural capacity of $N$. gonorrhoeae to suppress Th1and Th2-mediated specific immune responses against it and allow the development of protective immune responses that not only accelerate clearance of an existing infection (therapy) but also generate protection against future infection (prophylaxis). In our experiments so far, these include the systemic administration of blocking antibodies against TGF- $\beta$ and IL-10. Ongoing studies are aimed at determining whether these approaches can be developed into more practical interventions against genital tract infection with $N$. gonorrhoeae.

\section{METHODS}

Mice. All mice, including wild-type BALB/c and C57BL/6 mice, $\mathrm{C} 3 \mathrm{H} /$ $\mathrm{HeJ}$ (TLR4-deficient), C3H/FeJ (TLR4-normal), B6.129P2Il10tm1Cgn/J (IL-10 ${ }^{\mathrm{KO}}$ ), and B6.Cg-Tg(TcraTcrb)425Cbn/J (OT-II) mice on a C57BL/6 background, were purchased from Jackson Laboratories (Bar Harbor, ME). BALB/c mice were used for the experiments unless otherwise specified. Mice were maintained under standard conditions in the Laboratory Animal Facility at the University at Buffalo, Buffalo, NY, which is fully accredited by AAALAC. All animal use protocols were approved by the Institutional Animal Care and Use Committee of the University at Buffalo, Buffalo, NY.

Bacteria. N. gonorrhoeae FA1090 and an Opa-deletion mutant of FA1090 were kindly provided by Dr Janne Cannon (University of North Carolina at Chapel Hill). Bacteria were cultured on GC agar supplemented with hemoglobin and Isovitalex (BD Diagnostic Systems, Franklin Lakes, NJ) and the resultant growth was checked for colony morphology consistent with Opa protein and pilus expression. Bacteria were harvested from plates, and the cell density was determined as detailed previously. ${ }^{14}$

Mouse vaginal infection model. Female mice between 7 and 9 weeks old were infected with live N. gonorrhoeae FA1090 as previously described, ${ }^{14,39}$ with the modification that water-soluble estradiol was administered on days $-2,0$, and 2 . Vaginal mucus was quantitatively cultured daily on GC agar supplemented with selective antibiotics to determine the bacterial colonization loads. In some experiments, mice were injected subcutaneously on days $-1,0$, and every 2 days thereafter, with $300 \mu \mathrm{g}$ IL-10 or/and TGF- $\beta$-neutralizing monoclonal antibodies $(\mathrm{mAb})$ or control mouse IgG purchased from Bio X Cell (West Lebanon, NH).

Lymphocyte isolation and culture. Mononuclear cells were isolated from aseptically harvested ILN using Histopaque 1083 (SigmaAldrich, St Louis, MO) density-gradient centrifugation. CD4 ${ }^{+}$T cells were purified by magnetic-activated cell sorting (MACS) through negative selection using a Dynal CD4 cell isolation kit (Invitrogen, Carlsbad, CA). Cells were cultured in 24-well culture plates at a density of $1 \times 10^{6} \mathrm{cells} \mathrm{ml}^{-1}$ with either no stimulus or $N$. gonorrhoeae cells at indicated MOI. For some purified CD ${ }^{+} \mathrm{T}$-cell cultures, equal numbers of APC (mitomycin C-inactivated splenocytes) were added. $\mathrm{X}$-VIVO serum-free medium (Lonza, Walkersville, MD) with penicillin/streptomycin was used for all experiments. For some experiments, $40 \mu \mathrm{g} \mathrm{ml}^{-1}$ mouse IL-10 and/or TGF- $\beta$ neutralizing $\mathrm{mAb}$ (Bio X Cell) was added.

Th cell differentiation. Naive purified $\mathrm{CD}^{+} \mathrm{T}$ cells $(1 \times$ $10^{6}$ cells ml $^{-1}$ ) were cultured in 24 -well tissue culture plates and stimulated with $1.0 \mu \mathrm{g} \mathrm{ml}^{-1}$ plate-bound anti-CD3 antibody (eBiosciences, San Diego, CA) in complete X-VIVO medium. Differentiation of Th1, Th2, and Th17 cells was induced by the addition of IL- 12 and anti-IL- 4 antibody, IL- 4 and anti-IFN- $\gamma$ antibody, or TGF- $\beta$ and IL-23 plus anti-IFN- $\gamma$ and anti-IL- 4 antibodies, respectively, as detailed previously. ${ }^{15}$ In each condition, the cells were cultured in the absence or presence of $N$. gonorrhoeae FA1090 (MOI 50:1) and mitomycin C-inactivated APCs for 4 days. Supernatants and cells were then collected for ELISA and flow cytometry analyses. In some experiments, $40 \mu \mathrm{g} \mathrm{ml}^{-1}$ anti-IL-10 and/or anti-TGF- $\beta \mathrm{mAb}$ was added as indicated.

Vaginal explant culture and cell isolation. Genital tracts were dissected out aseptically and washed with Hanks's buffered salt solution. The tissue was manually separated into about $1 \mathrm{~mm}$ pieces, washed again, and weighed. Equal weights were added to each well of 24 -well cell culture plates. Tissue was incubated in X-VIVO serum-free 
medium supplemented with penicillin, streptomycin, and fungizone. Vaginal single-cell suspensions were prepared by protease digestion of excised vaginal tissue, as described previously. ${ }^{14}$

In vitro Tr1 cell induction. For generation of IL-10-induced Tr1 cells, $\mathrm{CD}^{+} / \mathrm{CD}^{+} 2 \mathrm{~L}^{+} \mathrm{T}$ cells were isolated from naive BALB/c mouse ILN by fluorescence-activated cell sorter (FACSAria; Becton-Dickinson, Franklin Lakes, NJ). The sorted cells were cultured and stimulated repeatedly for 3 weeks with anti-CD3 antibody (eBiosciences) in the presence or absence (control) of IL-10 $\left(10 \mathrm{ng} \mathrm{ml}^{-1}\right)$. Medium with antibodies, cytokines, and stimulants was changed twice a week, and IL-2 ( 50 units $\mathrm{ml}^{-1}$ ) was added to the cultures after 2 weeks. For generation of $N$. gonorrhoeae-induced $\operatorname{Tr} 1$ cells, ILN cells obtained from $N$. gonorrhoeae-infected mice were similarly stimulated for 3 weeks with anti-CD3 in the presence of N. gonorrhoeae. At the end of stimulation, $\mathrm{CD} 4{ }^{+}$cells were purified by MACS for adoptive transfer.

Cytokine ELISA. IL-10, TGF- $\beta$, IFN- $\gamma$, IL-12p70, IL-4, IL-5, and IL-17A levels were measured in triplicate using ELISA kits purchased from eBioscience.

Flow cytometry. Cells were washed with staining buffer twice, then incubated with the indicated antibodies for $30 \mathrm{~min}$ on ice, washed twice, and analyzed on a FACSCalibur cytometer (Becton-Dickinson). For intracellular staining, cells were first fixed with Cytofix/Cytoperm (eBioscience). Antibodies to mouse CD4, CD8, CD19, CD11b, CD11c, NKG2D, Gr-1, IFN- $\gamma$, IL-4, IL-17A, and Foxp3 conjugated with fluorescein isothiocyanate, phycoerythrin, or allophycocyanin were purchased from eBioscience. PE-conjugated anti-TGF- $\beta$ antibody was obtained from IQ Products (Groningen, The Netherlands).

Proliferation assays. Cells were labeled with carboxymethyl fluorescein succinimide ester (CFSE; Sigma-Aldrich) according to a previous protocol. ${ }^{40}$ CFSE-labeled cells were then washed twice in phosphate-buffered saline, recounted, and stimulated as described above. Cultured cells were harvested and then stained with allophycocyanin-conjugated anti-mouse CD4 antibody. The data were acquired by gating on the $\mathrm{CD} 4{ }^{+}$cell populations in a FACSCalibur cytometer. The sequential loss of CFSE fluorescence was used to measure cell proliferation.

Real-time RT-PCR. Total cellular RNA of MACS-purified, cultured $\mathrm{CD}^{+} \mathrm{T}$ cells or whole vaginas harvested from the mice was isolated with RNeasy Mini Kits (Qiagen, Valencia, CA) and was transcribed to cDNA using the iScript cDNA synthesis kit (Bio-Rad, Hercules, CA). Real-time RT-PCR was performed on an iCycler iQ detection system (Bio-Rad) using Sybergreen (Bio-Rad) for real-time monitoring of the PCR. The primers used were as follows: T-bet, $5^{\prime}$-GGAGCGGACCA ACAGCATCG-3'; 5'-GCGGGCGGCGGGAAGAACT-3'; GATA3, 5'-CCGAAACCGGAAGATGTCTA-3'; $5^{\prime}$-AGGGCTCTGCCTCTC TAACC-3'; ROR- $\gamma \mathrm{t}$, 5' ${ }^{\prime}$-CCGCTGAGAGGGCTTCAC-3' ${ }^{\prime} 5^{\prime}$-TGCAG GAGTAGGCCACATTAC- $3^{\prime}$; IFN- $\gamma, 5^{\prime}$-TACTGCCACGGCACAGT CATTGAA-3', 5'-GCAGCGACTCCTTTTCCGCTTCCT-3'; IL-4, $5^{\prime}$-GAAGCCCTACAGACGAGCTCA- $3^{\prime} ; 5^{\prime}$-ACAGGAGAAGGGAC GCCAT-3'; IL-17A, 5' -TCAGGGTCGAGAAGATGCTG-3'; $5^{\prime}$-TTT TCATTGTGGAGGGCAGA-3' ${ }^{\prime}$ IL-10, 5' -GCTCCTAGAGCTGCGG ACT- $3^{\prime} ; 5^{\prime}$ TGTTGTCCAGCTGGTCCTTT- $3^{\prime}$; and $\beta$-actin, $5^{\prime}$-CCTA AGGCCAACCGTGAAAAG- ${ }^{\prime}, 5^{\prime}$-GAGGCATACAGGGACAGCA CA- $3^{\prime}$. Relative quantification of target genes was analyzed based on the threshold cycle $(\mathrm{Ct})$ determined by Bio-Rad iQ5 optical system software.

Assay of serum and mucosal antibodies. Samples of saliva, vaginal wash, and serum were collected from individual mice on day 15 post inoculation. ${ }^{14}$ Gonococcus-specific IgA, IgG, and IgM in saliva, sera, and vaginal washes were measured by ELISA on plates coated with whole gonococci (FA1090). Total IgA, IgG, and IgM concentrations in secretions were assayed by ELISA on plates coated with anti-IgA, -IgG, or -IgM antibodies (Southern Biotech, Birmingham, AL). H5 mouse monoclonal antibody (specific for FA1090 porin; kindly provided by Ann E. Jerse, Uniformed Services University of the Health Sciences) or affinity-purified mouse IgA, IgG, and IgM (Southern Biotech) were used to establish standard curves. Bound antibodies were detected by alkaline phosphatase-conjugated goat anti-mouse IgA, IgG, or IgM antibody (Southern Biotech) and p-nitrophenylphosphate substrate (Southern Biotech). Plates were read in a VersaMax microplate reader (Molecular Devices, Sunnyvale, CA), and calibration curves for interpolation of unknowns were constructed using SoftMax software (Molecular Devices).

Statistical analysis. Data are expressed as the means \pm s.e.ms. Data on the effect of anti-IL-10 or/and anti-TGF- $\beta$ vs. control IgG treatments on mouse vaginal $N$. gonorrhoeae infection were analyzed using twoway analysis of variance with Fisher's protected least significant difference post hoc tests for multiple comparisons. Unpaired two-tailed $t$ tests were used to compare the mean values between two groups. $P<0.05$ was considered statistically significant.

SUPPLEMENTARY MATERIAL is linked to the online version of the paper at http://www.nature.com/mi

\section{ACKNOWLEDGEMENTS}

We are grateful to the assistance of the Confocal Microscope and Flow Cytometry Facility in the School of Medicine and Biomedical Sciences, University at Buffalo, Buffalo, NY, USA. We thank Dr Ann E. Jerse for helpful advice and valuable discussions during the course of this work. This work was supported by NIH grant Al074791 from the National Institute of Allergy and Infectious Diseases and by a grant from The John R. Oishei Foundation, Buffalo, NY, USA.

\section{DISCLOSURE}

The authors declared no conflict of interest.

c) 2014 Society for Mucosal Immunology

\section{REFERENCES}

1. World Health Organization Global Action Plan to Control the Spread and Impact of Antimicrobial Resistance in Neisseria gonorrhoeae. (WHO, Geneva, Switzerland, 2012).

2. Bolan, G.A., Sparling, P.F. \& Wasserheit, J.N. The emerging threat of untreatable gonococcal infection. N. Engl. J. Med. 366, 485-487 (2012).

3. Hook, E.W. \& Handsfield, H.H. Gonococcal infections in the adult. In Sexually Transmitted Diseases, 3rd edn, Holmes, K.K. \& Sparling, P.F. \& Mårdh, P.-A. \& Lemon, S.M. \& Stamm, W.E. \& Piot, P. \& Wasserheit, J.N., (eds) 451-466 (McGraw-Hill Health Professions Division, New York, NY, USA, 1999).

4. Cohen, M.S. Sexually transmitted diseases enhance HIV transmission: no longer a hypothesis. Lancet 351 (Suppl 3), 5-7 (1998).

5. Russell, M.W. \& Hook, E.W. 3rd Gonorrhea. In Vaccines for Biodefense and Emerging and Neglected Diseases (Barrett, A.D.T. \& Stanberry, L.R., eds) 963-981 (Academic Press, London, UK, , 2009).

6. Zhu, W. et al. Vaccines for gonorrhea: can we rise to the challenge?. Front. Microbiol. 2, 124 (2011).

7. Plummer, F.A. et al. Epidemiologic evidence for the development of serovar-specific immunity after gonococcal infection. J. Clin. Invest. 83, 1472-1476 (1989).

8. Ram, S. et al. Binding of C4b-binding protein to porin: a molecular mechanism of serum resistance of Neisseria gonorrhoeae. J. Exp. Med. 193, 281-295 (2001).

9. Smith, H., Parsons, N.J. \& Cole, J.A. Sialylation of neisserial lipopolysaccharide: a major influence on pathogenicity. Microb. Pathog. 19, 365-377 (1995).

10. Lewis, L.A., Burrowes, E., Rice, P.A. \& Ram, S. Interactions of Neisseria with complement. In Neisseria: Molecular Mechanisms of Pathogenesis (Genco, C.A. \& Wetzler, L., eds) 123-144 (Caister Academic Press, Norfolk, UK, 2010). 
11. Boulton, I.C. \& Gray-Owen, S.D. Neisserial binding to CEACAM1 arrests the activation and proliferation of CD4 + T lymphocytes. Nat. Immunol. $\mathbf{3}$, 229-236 (2002).

12. Zhu, W. et al. Neisseria gonorrhoeae suppresses dendritic cell-induced, antigen-dependent CD4 T cell proliferation. PLoS One 7, e41260 (2012).

13. Feinen, B., Jerse, A.E., Gaffen, S.L. \& Russell, M.W. Critical role of Th17 responses in a murine model of Neisseria gonorrhoeae genital infection. Mucosal Immunol. 3, 312-321 (2010).

14. Liu, Y. \& Russell, M.W. Diversion of the immune response to Neisseria gonorrhoeae from Th17 to Th1/Th2 by treatment with anti-transforming growth factor beta antibody generates immunological memory and protective immunity. mBio 2, e00095-11 (2011).

15. Liu, Y. et al. Neisseria gonorrhoeae selectively suppresses the development of Th1 and Th2 cells, and enhances Th17 cell responses, through TGF-beta-dependent mechanisms. Mucosal Immunol. 5, 320-331 (2012).

16. Sabat, R. et al. Biology of interleukin-10. Cytokine Growth Factor Rev. 21, 331-344 (2010).

17. Ouyang, W., Rutz, S., Crellin, N.K., Valdez, P.A. \& Hymowitz, S.G. Regulation and functions of the IL-10 family of cytokines in inflammation and disease. Annu. Rev. Immunol. 29, 71-109 (2011).

18. Roncarolo, M.G. et al. Interleukin-10-secreting type 1 regulatory T cells in rodents and humans. Immunol. Rev. 212, 28-50 (2006).

19. Battaglia, M., Gregori, S., Bacchetta, R. \& Roncarolo, M.G. Tr1 cells: from discovery to their clinical application. Semin. Immunol. 18, 120-127 (2006).

20. Kreisman, L.S. \& Cobb, B.A. Glycoantigens induce human peripheral $\operatorname{Tr} 1$ cell differentiation with gut-homing specialization. J. Biol. Chem. 286, 8810-8818 (2011).

21. Gianfrani, C. et al. Gliadin-specific type 1 regulatory T cells from the intestinal mucosa of treated celiac patients inhibit pathogenic $T$ cells. J. Immunol. 177, 4178-4186 (2006).

22. McGuirk, P., McCann, C. \& Mills, K.H. Pathogen-specific T regulatory 1 cells induced in the respiratory tract by a bacterial molecule that stimulates interleukin 10 production by dendritic cells: a novel strategy for evasion of protective Thelper type 1 responses by Bordetella pertussis. J. Exp. Med. 195, 221-231 (2002).

23. Kobayashi, R. et al. Induction of IL-10-producing CD4 + T-cells in chronic periodontitis. J. Dent. Res. 90, 653-658 (2010).

24. Cole, J.G., Fulcher, N.B. \& Jerse, A.E. Opacity proteins increase Neisseria gonorrhoeae fitness in the female genital tract due to a factor under ovarian control. Infect. Immun. 78, 1629-1641 (2010).

25. Packiam, M. et al. Protective role of Toll-like receptor 4 in experimental gonococcal infection of female mice. Mucosal Immunol. 5, 19-29 (2012).

26. John, C.M., Liu, M. \& Jarvis, G.A. Profiles of structural heterogeneity in native lipooligosaccharides of Neisseria and cytokine induction. J. Lipid Res. 50, 424-438 (2009).
27. Imarai, $M$. et al. Regulatory $T$ cells are locally induced during intravaginal infection of mice with Neisseria gonorrhoeae. Infect. Immun. 76, 5456-5465 (2008).

28. Huber, S. et al. Th17 cells express interleukin-10 receptor and are controlled by Foxp3(-) and Foxp3 + regulatory CD4 + T cells in an interleukin-10-dependent manner. Immunity 34, 554-565 (2011).

29. Feinen, B. \& Russell, M.W. Contrasting roles of IL-22 and IL-17 in murine genital tract infection by Neisseria gonorrhoeae. Front. Immunol. 3, 11 (2012).

30. Song, W. et al. Local and humoral immune responses against primary and repeat Neisseria gonorrhoeae genital tract infections of 17 beta-estradioltreated mice. Vaccine 26, 5741-5751 (2008).

31. Liu, Y., Feinen, B. \& Russell, M.W. New concepts in immunity to Neisseria gonorrhoeae: innate responses and suppression of adaptive immunity favor the pathogen, not the host. Front. Microbiol. 2, 52 (2011).

32. Criss, A.K. \& Seifert, H.S. Neisseria gonorrhoeae suppresses the oxidative burst of human polymorphonuclear leukocytes. Cell. Microbiol. 10, 2257-2270 (2008).

33. Criss, A.K., Katz, B.Z. \& Seifert, H.S. Resistance of Neisseria gonorrhoeae to non-oxidative killing by adherent human polymorphonuclear leucocytes. Cell. Microbiol. 11, 1074-1087 (2009).

34. Shafer, W.M., Qu, X., Waring, A.J. \& Lehrer, R.I. Modulation of Neisseria gonorrhoeae susceptibility to vertebrate antibacterial peptides due to a member of the resistance/nodulation/division efflux pump family. Proc. Natl. Acad. Sci. USA 95, 1829-1833 (1998).

35. Packiam, M., Veit, S.J., Anderson, D.J., Ingalls, R.R. \& Jerse, A.E. Mouse strain-dependent differences in susceptibility to Neisseria gonorrhoeae infection and induction of innate immune responses. Infect. Immun. 78, 433-440 (2010).

36. Gagliardi, M.C. et al. Circulating levels of interleukin-17A and interleukin-23 are increased in patients with gonococcal infection. FEMS Immunol. Med. Microbiol. 61, 129-132 (2011).

37. Pantelic, M. et al. Neisseria gonorrhoeae kills carcinoembryonic antigen-related cellular adhesion molecule 1 (CD66a)-expressing human B cells and inhibits antibody production. Infect. Immun. 73, 4171-4179 (2005).

38. Sparling, P.F., Elkins, C., Wyrick, P.B. \& Cohen, M.S. Vaccines for bacterial sexually transmitted infections: a realistic goal?. Proc. Natl. Acad. Sci. USA 91, 2456-2463 (1994).

39. Jerse, A.E. Experimental gonococcal genital tract infection and opacity protein expression in estradiol-treated mice. Infect. Immun. 67, 5699-5708 (1999).

40. Chen, J.C., Chang, M.L. \& Muench, M.O. A kinetic study of the murine mixed lymphocyte reaction by 5,6-carboxyfluorescein diacetate succinimidyl ester labeling. J. Immunol. Methods 279, 123-133 (2003). 